\title{
Telemedizin in der Onkologie: Qualität verbessern - aber wie?
}

\author{
Henning Adam, Annette Lebeau, Andreas Turzynski, Verena Materna, \\ Stefan Rakowsky und Simone Wesselmann
}

(c) Der/die Autor(en) 2019

J. Klauber et al. (Hrsg.), Krankenhaus-Report 2019

https://doi.org/10.1007/978-3-662-58225-1_11

\section{Zusammenfassung}

Telemedizin, definiert als ärztliche Leistungserbringung über räumliche oder zeitliche Distanz hinweg und unter Einsatz moderner Informations- und Kommunikationstechnologien, gewinnt auch in der Versorgung von Krebspatienten an Bedeutung. Die onkologische Versorgung in Deutschland wird maßgeblich von den zertifizierten Zentren der Deutschen Krebsgesellschaft geprägt. Die Zentren erfüllen hohe Qualitätsanforderungen, beispielsweise mit Blick auf die fachliche Expertise und interdisziplinäre Zusammenarbeit. Der so bestehende große Kommunikationsbedarf eröffnet potenzielle Einsatzfelder für die Telemedizin. Anhand der Beispiele Tele-Tumorkonferenz und Telepathologie beschreibt dieser Beitrag Chancen und Risiken bei der Implementierung von Telemedizin in der Onkologie: Sie ermöglicht den Leistungserbringern eine effizientere Zusammenarbeit und die flächendeckende Einbringung onkologischen Wissens. Andererseits besteht je nach Einsatzbereich und Umsetzung die Gefahr von Informationsverlusten oder Einbußen für den Versorgungsstandard. Diese Aspekte gilt es kritisch abzuwägen, damit die Telemedizin die Onkologie positiv weiterentwickelt und gleichzeitig die Qualität der Patientenversorgung erhalten bleibt.

Telemedicine, defined as the provision of medical services at a geographical or temporal distance and using modern information and communication technologies, is getting more and more important in the care of cancer patients. The certified centres of the German Cancer Society play a decisive role in oncological care in Germany. The centres meet high quality standards, for example with regard to specialist expertise and interdisciplinary cooperation. The resulting great need for communication creates potential fields of application for telemedicine. Using the examples of tele-tumour conference and telepathology, this article describes the opportunities and risks associated with the implementation of telemedicine in oncology: It enables service providers to cooperate more efficiently and to contribute oncological knowledge across the board. On the other hand, depending on the area of application and implementation, there is a risk of information being lost or the standard of care being compromised. These aspects need to be carefully considered so that oncology can be positively developed by telemedicine and, at the same time, the quality of patient care is maintained.

\subsection{Einleitung}

Über 90-mal enthält der im Jahr 2018 zwischen CDU, CSU und SPD geschlossene Koalitionsvertrag für die 19. Legislaturperiode der Bundesregierung das Wort „Digitalisierung“. Bereits diese Zahl, aber ebenfalls die im Vertrag vereinbarten Ziele und Maßnahmen bringen zum Ausdruck, dass sich unsere Gesellschaft in einem digitalen Wandel befindet, der seitens der Politik gefordert und gefördert wird. 
Auch das Gesundheitswesen sieht sich mit der Digitalisierung und den mit ihr verbundenen Chancen und Risiken konfrontiert. Immer mehr Gesundheits-Apps kommen auf den Markt, die elektronische Gesundheitskarte wurde eingeführt und soll in ihren Funktionen weiter ausgebaut werden und die medizinischen Berufsgruppen kommunizieren zunehmend unter Einsatz moderner Informations- und Kommunikationstechnologien (IKT). Die Telemedizin, verstanden als ärztliche Leistungserbringung unter Anwendung von IKT über räumliche oder zeitliche Distanz, ist ein Teilbereich der Digitalisierung im Gesundheitswesen. Sie ist bereits heute Versorgungsrealität in Deutschland und wird je nach Fachdisziplin und Versorgungskontext mit unterschiedlicher Intensität genutzt. Ziel des Einsatzes von Telemedizin ist es, die Gesundheitsversorgung effizienter zu gestalten und Versorgungslücken, beispielsweise in unterversorgten Regionen, zu schließen.

Die Onkologie ist ein Versorgungsbereich, der das Gesundheitswesen heute und in Zukunft vor große Herausforderungen stellt. Jährlich erkranken über 450.000 Menschen in Deutschland an Krebs (Robert Koch-Institut 2017). Krebserkrankungen sind nach den Herz-Kreislauf-Erkrankungen die zweithäufigste Todesursache in Deutschland, sie führen zu hohen Gesundheitsausgaben und bestimmen einen wichtigen Teil der Krankheitslast der deutschen Bevölkerung. Ganz automatisch stellt sich die Frage, ob und wie die Telemedizin in die Versorgung von Krebspatienten sinnstiftend eingebracht werden kann. Die Krebsversorgung in Deutschland wird zu einem gewichtigen Anteil von den zertifizierten Zentren der DKG gestaltet. Deren Prinzip einer qualitätsgesicherten, leitliniengetreuen und interdisziplinären Patientenversorgung bietet verschiedenste Einsatzmöglichkeiten für die Telemedizin. Dieser Artikel beschreibt anhand von zwei konkreten Beispielen - Tumorkonferenzen und Pathologie - Chancen und Risiken bei der Implementierung telemedizinischer Versorgungskonzepte und diskutiert darauf aufbauend, unter welchen Bedingungen Telemedizin die onkologische Versorgung wirksam unterstützen kann.

\subsection{Telemedizin: Definition und Verbreitung in Deutschland}

Eine allgemein anerkannte Definition für die Telemedizin existiert nicht. Unter dem Oberbegriff der Digitalisierung im Gesundheitswesen ist die Telemedizin in eine Systematik mit weiteren Begriffen wie eHealth, Gesundheitstelematik oder mHealth eingebettet, die sich teilweise überschneiden. Sie beschreiben alle die Anwendung moderner IKT zum Ziele der Verbesserung der Gesundheit und Unterstützung der Gesundheitsversorgung (Bundesministerium für Wirtschaft und Energie 2016). Im Gegensatz zu den globaleren Begriffen wie eHealth oder Gesundheitstelematik fokussiert die Telemedizin dabei auf digitale Versorgungskonzepte zur Erbringung ärztlicher Leistungen.

Der 113. Ärztetag hat 2015 die Telemedizin definiert als einen

„Sammelbegriff für verschiedenartige ärztliche Versorgungskonzepte, die als Gemeinsamkeit den prinzipiellen Ansatz aufweisen, dass medizinische Leistungen der Gesundheitsversorgung der Bevölkerung in den Bereichen Diagnostik, Therapie und Rehabilitation sowie bei der ärztlichen Entscheidungsberatung über räumliche Entfernungen (oder zeitlichen Versatz) hinweg erbracht werden. Hierbei werden Informations- und Kommunikationstechnologien eingesetzt." (Bundesärztekammer 2015)

Der Einsatz telemedizinischer Versorgungskonzepte wird auf gesundheitspolitischer Ebene seit vielen Jahren diskutiert. Insgesamt ist die Telemedizin mit der Erwartung verbunden, die ärztliche Kommunikation inter- und intrasektoral zu verbessern und in Zeiten zunehmenden Ärztemangels und vor dem Hintergrund des demografischen Wandels eine flächendeckende, qualitativ hochwertige Gesundheitsversorgung sicherzustellen. Es lassen sich unterschiedliche Anwendungsarten der Telemedizin abgrenzen, die entweder eine Arzt-zuArzt- oder Arzt-zu-Patient-Kommunikation zum Gegenstand haben. Beispiele für Anwendungsarten sind die Telekonsultation, das Telemonitoring oder die Teletherapie (Fischer et al. 2016; Marx und Beckers 2015).

Mit dem zum 1. Januar 2016 in Kraft getretenen "Gesetz für sichere digitale Kommunikation und Anwendungen im Gesundheitswesen" (E-Health- 
Gesetz) wurden die gesetzlichen Rahmenbedingungen zum Ausbau der Telematikinfrastruktur zuletzt angepasst (Deutscher Bundestag 2015). Als einen Meilenstein verankerte der Gesetzgeber, dass bis Ende 2018 die Voraussetzungen zur Nutzung einer elektronischen Patientenakte geschaffen werden. Aber auch der Ausbau und die Verknüpfung telemedizinischer Versorgungskonzepte sollte mit dem Gesetz weiter gefördert werden, beispielsweise durch ein öffentlich zugängliches Interoperabilitätsverzeichnis. Dieses soll bundesweite Telemedizin-Projekte auflisten und deren verwendete informationstechnische Standards beschreiben.

Die nach aktuellem Stand im Interoperabilitätsverzeichnis gelisteten 168 Projekte zeigen auf, dass bereits heute telemedizinische Versorgungsstrukturen in Deutschland etabliert sind (Vesta Informationsportal 2018). In besonderem Maße telemedizinisch ausgebaute Versorgungsbereiche sind die Radiologie und die Schlaganfallversorgung (Schenkel 2017; Breuer und Schwab 2017). In der Regel handelt es sich um örtliche oder regionale Versorgungsansätze.

Trotz der bereits bestehenden breiten Anwendung von Telemedizin in einigen Bereichen werden aber auch immer wieder Herausforderungen und Hürden bei der Implementierung telemedizinischer Versorgung beschrieben. Hierzu gehören technische Schwierigkeiten, fehlende Vergütungsoptionen, Vorbehalte und Skepsis seitens der Ärzte und Patienten sowie juristische Fragestellungen (Fischer et al. 2016). Rechtlich steht neben Datenschutz- und Haftungsfragen dabei auch immer das in der ärztlichen Berufsordnung verankerte Fernbehandlungsverbot im Mittelpunkt. Letzteres wurde 2018 durch eine Änderung der Musterberufsordnung für Ärzte gelockert, sodass unter bestimmten Voraussetzungen nunmehr auch ausschließlich auf Kommunikationsmedien beruhende Patientenbehandlungen möglich sind (Bundesärztekammer 2018).

\subsection{Onkologische Versorgung und Zertifizierung}

Die onkologische Versorgung in Deutschland wird maßgeblich durch das Zertifizierungssystem der Deutschen Krebsgesellschaft (DKG) geprägt. Das
System wurde 2003 mit dem Ziel einer qualitätsgesicherten und umfassenden Versorgung von Krebspatienten entwickelt und seitdem kontinuierlich ausgebaut (Wesselmann 2012). Heute werden etwa 40 Prozent der jährlichen Krebsneuerkrankungen in einem DKG-zertifizierten Zentrum behandelt. Das Zertifizierungssystem deckt alle Krebsarten ab und für viele Tumorentitäten, darunter die am häufigsten vorkommenden Tumorerkrankungen Brust-, Darm-, Lungen- und Prostatakrebs, werden tumorspezifische Zertifikate vergeben. An über $420 \mathrm{Kran}$ kenhäusern sind deutschlandweit zertifizierte Zentren etabliert.

Das Zertifizierungssystem ist integraler Bestandteil des Qualitätszyklus in der Onkologie, der 2008 im Nationalen Krebsplan festgeschrieben wurde (Mensah et al. 2017). Ausgangspunkt des Qualitätszyklus ist die Erstellung evidenzbasierter Leitlinien. Diese bilden die Grundlage, um in den Kommissionen des Zertifizierungssystems die Anforderungen an die Struktur-, Prozess- und Ergebnisqualität für die Zertifizierung onkologischer Leistungserbringer, der sogenannten Zentren, festzulegen. Von den Kommissionen unabhängige Auditoren prüfen in den Zentren vor Ort, ob die Anwärter für ein Zertifikat die festgelegten Anforderungen erfüllen. Die Versorgungsqualität der zertifizierten Zentren wird anhand definierter Qualitätsindikatoren erhoben und in jährlichen Berichten transparent veröffentlicht. Über die Jahresberichte schließt sich dann der onkologische Qualitätszyklus. Die gemessenen Qualitätszahlen ermöglichen, in den Zertifizierungskommissionen und Leitliniengruppen über die Weiterentwicklung der Anforderungen zu beraten und in den Zentren die Anpassung von Strukturen oder Prozessen zur besseren Erfüllung der Qualitätsvorgaben anzustoßen.

Das Zertifizierungssystem ist nicht nur darauf ausgerichtet, eine leitliniengerechte Behandlung sicherzustellen. Es soll auch die interdisziplinäre und multiprofessionelle Zusammenarbeit fördern. Dies wird dadurch erreicht, dass die Zentren für die Zertifizierung ein Netzwerk aus unterschiedlichen ärztlichen Fachrichtungen und weiteren medizinischen Berufsgruppen sektorenübergreifend nachweisen müssen (Kowalski et al. 2017). Zu den Hauptbehandlungspartnern des Netzwerks zählen neben der tumorspezifischen chirurgischen und konser- 


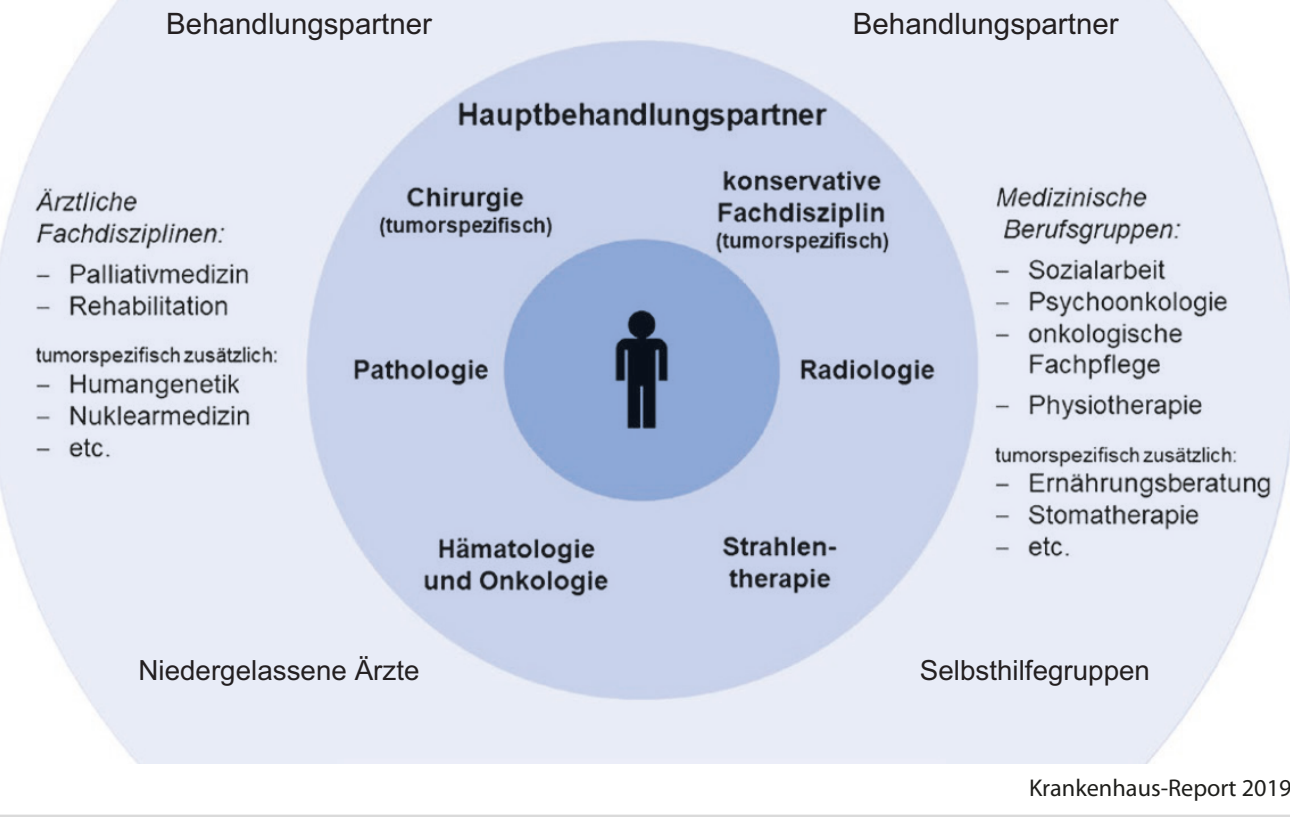

- Abb. 11.1 Struktur eines zertifizierten Zentrums

vativen Fachdisziplin auch immer die Strahlentherapie, Pathologie, Radiologie sowie Hämatologie und Onkologie. Zudem ist die Zusammenarbeit mit weiteren ärztlichen Fachrichtungen (z. B. der Palliativmedizin, Humangenetik oder Nuklearmedizin) erforderlich. Weitere medizinische Berufsgruppen wie die Psychoonkologie, Sozialarbeit und Physiotherapie müssen für die Patientenversorgung verfügbar sein und das Zentrum muss mit Selbsthilfegruppen und niedergelassenen Ärzten kooperieren (- Abb. 11.1). Die Sicherstellung der Kommunikation und Zusammenarbeit im zertifizierten Netzwerk erfolgt auf der Basis von Kooperationsverträgen, Fortbildungen und Qualitätszirkeln. Das wichtigste Strukturelement des interdisziplinären Austauschs ist die Tumorkonferenz. In dieser treffen sich die unterschiedlichen Behandlungspartner des Zentrums, um die Patientenfälle zu diskutieren und gemeinschaftlich zu einer Therapieempfehlung für die Patienten zu kommen.

Das so strukturierte Zertifizierungssystem trägt zu einer Verbesserung der Versorgungsqualität für Krebspatienten bei. Dies lässt sich anhand aktueller Studienergebnisse belegen, die verbesserte Über- lebensraten für die in zertifizierten Zentren behandelte Patienten berichten (Völkel et al. 2018; Trautmann et al. 2018).

\subsection{Telemedizinischer Versorgungs- bedarf in der Onkologie}

Telemedizinische Versorgungsansätze sind auch für die Onkologie und das Zertifizierungssystem von großer Relevanz. Denn für die Patientenversorgung in den zertifizierten Zentren ist unterschiedlichste fachlich-medizinische Expertise und ein hohes Maß an interdisziplinärer Kommunikation erforderlich. Hier kann die Telemedizin in der Strukturierung und Prozessplanung der zertifizierten Zentren einen unterstützenden Beitrag leisten. Anhand von zwei konkreten Beispielen wird in den folgenden Abschnitten die Umsetzung telemedizinischer Versorgungskonzepte in der Onkologie dargestellt.

Die Tumorkonferenz steht als wichtigstes Strukturelement für den interdisziplinären Austausch im zertifizierten Zentrum im besonderen Fokus telemedizinischer Lösungsansätze. Bereits heute wird 
die Durchführung telemedizinischer Tumorkonferenzen vielerorts praktiziert (Janssen et al. 2017; Güttler et al. 2012) und auch die Anforderungen an die zertifizierten Zentren sehen die telemedizinische interdisziplinäre Fallbesprechung explizit als eine Option vor. Am Beispiel des Charité Comprehensive Cancer Center (CCCC) werden daher im - Abschn. 11.5 die Chancen und Herausforderungen bei der Implementierung einer telemedizinischen Tumorkonferenz beschrieben.

Die Begutachtung histologischer Präparate ist ein weichenstellender Prozessschritt für die Diagnostik und Therapieplanung bei allen Tumorerkrankungen. Daher ist die Pathologie ein unverzichtbarer Bestandteil aller zertifizierten Zentren. Um eine flächendeckende Verfügbarkeit der pathologischen Expertise in Deutschland langfristig zu garantieren, werden in den letzten Jahren zunehmend telepathologische Versorgungskonzepte diskutiert. Die technische Umsetzung solcher Konzepte unter Gewährleistung einer qualitativ hochwertigen pathologischen Befundung ist jedoch herausfordernd. Daher wird der $>$ Abschn. 11.6 die Diskussion um die telepathologische onkologische Versorgung aufgreifen.

\subsection{Die Telemedizinische Tumorkon- ferenz am Beispiel des Charité Comprehensive Cancer Centers}

\subsubsection{Struktur des Zentrums}

Das CCCC ist das integrative Onkologische Zentrum der Charité - Universitätsmedizin Berlin, in dem alle Kliniken, Institute der Grundlagenforschung und diagnostischen Institute mit Bezug zur Krebsmedizin organisiert sind. Es wurde 2008 gegründet und wird seitdem beständig ausgebaut. Die beteiligten Bereiche arbeiten gemäß der Satzung des CCCC zusammen. Übergeordnete Entscheidungen werden im Lenkungsausschuss gemeinsam getroffen. Die einzelnen Organ- und Querschnittbereiche des Onkologischen Zentrums sind nach den Anforderungen der DKG zertifiziert und werden jährlich hinsichtlich der Einhaltung der Qualitätskriterien auditiert. Darüber hinaus ist das CCCC eines von derzeit 13 Onkologischen Spitzenzentren (Stand:
August 2018), die von der Deutschen Krebshilfe e. V. gefördert werden und sich regelmäßig in den verschiedenen Arbeitsgruppen des CCCC-Netzwerks austauschen, um die Standards in der Krebsmedizin weiterzuentwickeln. Das CCCC ist regional und überregional stark vernetzt, u. a. mit ärztlichen und wissenschaftlichen Kolleginnen und Kollegen anderer Kliniken und im niedergelassenen Bereich.

\subsubsection{Strukturelle Besonderheiten}

Das CCCC ist primär an drei verschiedenen KlinikStandorten aktiv, um innerhalb Berlins den Patientinnen und Patienten flächendeckend eine interdisziplinäre und fortschrittliche onkologische Versorgung anbieten zu können - am Charité Campus Mitte (CCM), am Campus Virchow-Klinikum im Bezirk Wedding (CVK) und am Campus Benjamin Franklin im Bezirk Steglitz (CBF). Von der Diagnosestellung über die Therapie bis hin zur Nachsorge arbeiten die verschiedenen Fachabteilungen der Charité campusübergreifend eng zusammen, sowohl im Rahmen der leitlinienkonformen Versorgung nach den evidenzbasierten Standards als auch bei der Planung und Durchführung von Studien für die Entwicklung neuer diagnostischer und therapeutischer Verfahren. Um den standortübergreifenden Austausch der beteiligten Partner zu den verschiedensten Themen zu optimieren, werden zunehmend Videokonferenzschaltungen etabliert, die bei den campusübergreifenden Tumorkonferenzen bereits seit einigen Jahren an der Charité standardmäßig genutzt werden (Güttler et al. 2012).

\subsubsection{Organisation und Ablauf der telemedizinischen Tumor- konferenz}

Die Tumorkonferenz-Teams sind gemäß den medizinischen Anforderungen zusammengesetzt und entsprechen den Anforderungen der DKG. Vertreten sind hier in der Regel mindestens eine chirurgische Abteilung, das entitätsspezifische internistische Fachgebiet, die Hämatologie und Onkologie, Radiologie, Strahlentherapie (ggf. auch die Nuklearmedizin) und die Pathologie. 


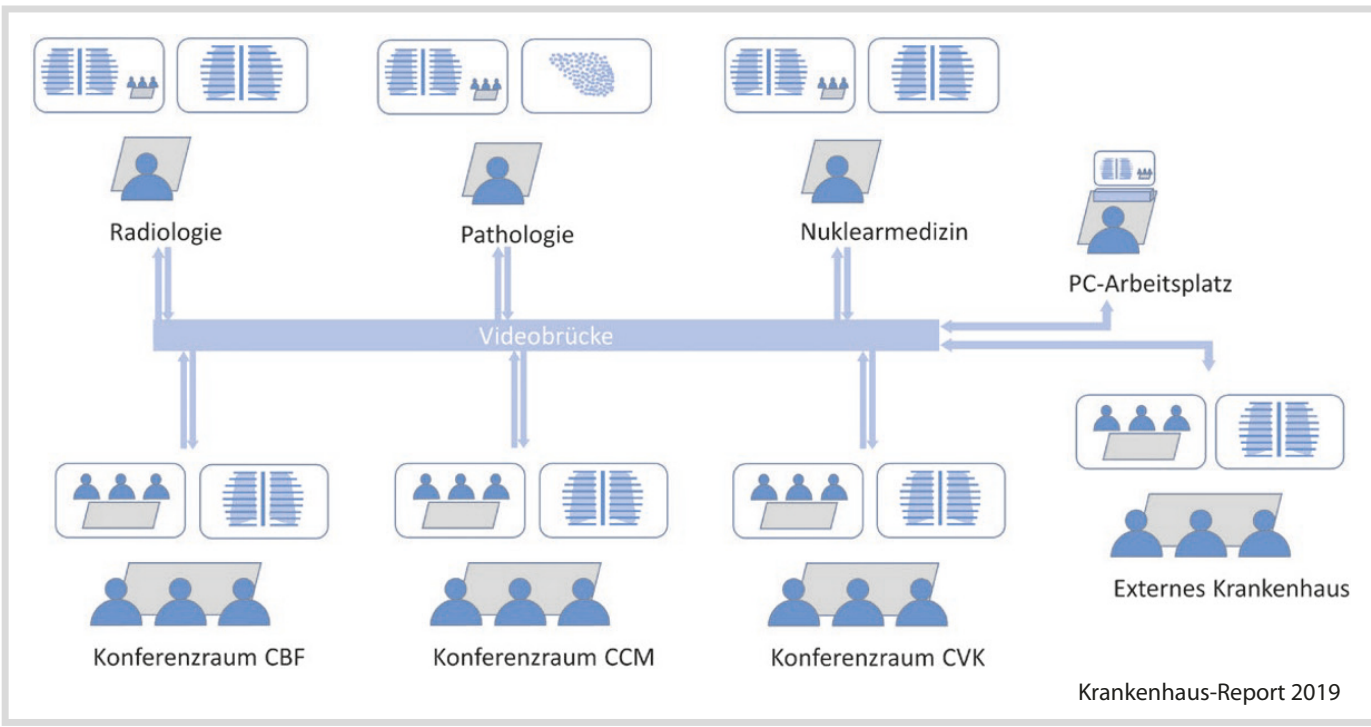

Abb. 11.2 Televideokonferenz-Plattform für die standortübergreifende Vernetzung und Integration der Diagnostikabteilungen in Tele-Tumorkonferenzen

Im Vorfeld der Tumorkonferenz erfolgt die Anmeldung der zu besprechenden Patienten im zentralen Anmeldeportal (= WebGTDS, web-basierte Oberfläche des Gießener Tumordokumentationssystems) durch die vorstellenden Ärzte unter Beschreibung der bisherigen Befunde und Nennung der Fragestellung. Anhand der Anmeldungen bereiten sich die Fachdisziplinen auf die Tumorkonferenz vor. Zum Abschluss der Besprechung eines Patienten wird die Empfehlung der Tumorkonferenz im WebGTDS dokumentiert und anschließend in das Krankenhausinformationssystem übertragen. Der Tumorkonferenz-Koordinator des CCCC unterstützt die ärztlichen Kollegen bei den administrativen Prozessen, u. a. bei der Einrichtung von Anmeldeportal-Zugängen inklusive Berechtigungen sowie bei der Nutzung des Videokonferenzsystems in den eingerichteten Videokonferenzräumen.

Aufgrund der engen campusübergreifenden Zusammenarbeit werden Tumorkonferenzen als standortübergreifende Tele-Tumorkonferenz durchgeführt. Beispielsweise finden sich bei einer 2-Standort-Tele-Tumorkonferenz die Vertreter der Kliniken am Standort A im dortigen Konferenzraum zusammen und können sich in die gewünschte Telekonferenz einwählen, in die sich auch die Teilnehmer im Konferenzraum am Standort B einge- wählt haben. Die Diagnostikabteilungen Radiologie, Pathologie und Nuklearmedizin schalten sich ebenfalls von lokalen telemedizinischen Arbeitsplätzen zu (• Abb. 11.2). Bei Bedarf steht die Zuschaltung externer Krankenhäuser und niedergelassener Ärzte zur Vorstellung der dort behandelten Patienten zur Verfügung.

In den Konferenzräumen sind in der Regel zwei Monitore vorhanden, auf denen die zugeschalteten Teilnehmer sowie die Patientendaten aus dem WebGTDS zu sehen sind. Während der Vorstellung des Patienten wird zu den radiologischen bzw. den histologischen Bildern umgeschaltet. Alle Verschaltungen können die Nutzer selbstständig ausführen, jedoch steht auch ein CCCC-Mitarbeiter im zentralen CCCC-Telekonferenzraum zur Unterstützung bereit.

\subsubsection{Implementierungsprozess der telemedizinischen Tumor- konferenz}

Die Möglichkeit der Unterstützung von Tumorkonferenzen durch Televideokonferenzen ist ausdrücklich in den Anforderungen für die DKG-Zertifizierung vorgesehen unter der Voraussetzung, dass alle 
Teilnehmer die Patientendaten und -bilder in ausreichender Qualität sehen können.

Am CCCC wurden hierfür in das CharitéDatennetzwerk integrierte High-Definition-Videokonferenzsysteme für die gleichzeitige Übertragung von Kamerabild und Daten in den Konferenzräumen installiert. Die Videoausgabe erfolgt auf zwei großflächigen Monitoren (je nach Raumgröße 50-75 Zoll Diagonale). Für die Audioübertragung in HD-Audio-Qualität wird die zugehörige integrierte Mikrofon-Lautsprecher-Kombination bzw. professionelle Audiotechnik verwendet. Touchscreen-Menüs und Mediensteuerungen erleichtern eine intuitive Bedienung der Videokonferenzsysteme durch die Nutzer. Eine zentrale Videobrücke der Charité ermöglicht das Zusammenschalten mehrerer Standorte.

Besonderer Wert wird auf die Übertragung der radiologischen Bilder gelegt. Die Ausrichtung der Grafikausgabe des radiologischen Befundungsarbeitsplatzes muss von Hochformat auf Querformat geändert werden, damit sie als sekundärer Videostrom im Standard des Videokonferenzsystems übertragen werden kann. Seit kurzem werden in den teleradiologischen Räumen auch Arbeitsstationen mit Monitoren mit Full-HD-Auflösung im Querformat genutzt, die ohne Anpassung vom Videokonferenzsystem übertragen werden können. Das live Durchblättern durch die Schnittbilder wird ohne Verzögerung übertragen, indem der Anteil der Bandbreite für den Datenkanal erhöht wird.

Die Datensicherheit ist bei den Charité-internen Telekonferenzen im durch eine Firewall geschützten Charité-Intranet sowie die Verwendung einer verschlüsselten Übertragung bei externen Verbindungen im Internet gegeben. Es werden keine externen Videokonferenzdienste in einer Cloud genutzt, sondern die Charité-interne Videobrücke.

\subsubsection{Vor- und Nachteile}

Die Tele-Tumorkonferenzen sind ein Anwendungsfeld der Unterstützung der Arzt-zu-Arzt-Interaktion durch Telemedizin. Die resultierende Zeitersparnis und Steigerung der Effizienz und Qualität ermöglichen erst die routinemäßige standortübergreifende Zusammenarbeit.
Ebenso unterstützen Telekonferenzen den Aufbau eines regionalen Netzwerks durch die Anbindung anderer Krankenhäuser und niedergelassener Ärzte an das CCCC für die Patientenversorgung und den Wissenstransfer.

Für die technische Realisierung wurden kommerzielle Standard-Videokonferenzsysteme verwendet, die eine Interoperabilität mit gängigen Systemen auch anderer Kliniken gewährleisten. Ebenso ist diese Implementierung flexibel bezüglich sich ändernder Anforderungen an die Konferenzabläufe und die Zusammensetzung der Teilnehmer. Die Verschaltungsvorgänge müssen an die Möglichkeiten der Videokonferenzsysteme ausgerichtet werden. Perspektivisch wird eine Standardisierung der Videokonferenzräume an der Charité angestrebt. Die Übertragung des Raumkamerabilds aus den Konferenzräumen ist gegenüber der von der DKG mindestens geforderten Übertragung von Audio und vorgestellten Unterlagen/Bildmaterial ein Plus, das die Telekommunikation erleichtert und im CCCC nicht mehr wegzudenken ist.

Generell erfordert Televideokommunikation eine gewisse Disziplin der Teilnehmer, damit die Kommunikation sehr natürlich und direkt erfolgt und als Telepräsenz bezeichnet werden kann. So sollte jeweils nur ein Teilnehmer sprechen, störende Nebengeräusche und Telefonate sollten vermieden werden und auf Nutzung der Mikrofone und Einschalten der Beleuchtung sollte geachtet werden.

Die relativ hohen Kosten für die Ausstattung eines Videokonferenzraums werden durch die Nutzungsfrequenz ( $>20$ wöchentliche Tele-Tumorkonferenzen) und Synergien durch andere Nutzung der Videokonferenztechnik an der Charité aufgewogen. Investitionen in neue State-of-the-Art-Videokonferenzsysteme sind einzuplanen, um die Anwendung weiterzuentwickeln und die Nutzerfreundlichkeit weiter zu verbessern.

Auf dem aktuellen Wochenplan der Tumorkonferenzen am CCCC stehen 25 wöchentliche TeleTumorkonferenzen mit einer Gesamtdauer von 32 Stunden pro Woche. Durchschnittlich nehmen acht Fachärzte (zwischen minimal drei und maximal 14) pro Konferenz als Spezialisten an den Tumorkonferenzen teil, insgesamt ca. 200 Fachärzte pro Woche. In den Tumorkonferenzen wurden im Jahr 2017 insgesamt 9.300 Patienten besprochen. 
Diese Zahlen belegen, dass die Organisationsform der Tumorkonferenzen als standortübergreifende Televideokonferenz für ein sehr großes Zentrum effizient funktioniert. Darüber hinaus werden durch die Tele-Tumorkonferenzen die Einhaltung diagnostischer Standards und die Durchführung von standortübergreifenden Studien gefördert.

\subsection{Telepathologie in der Onkologie}

\subsubsection{Pathologieversorgung in Deutschland}

Von den rund 1.950 Krankenhäusern in Deutschland verfügen nur noch etwa 150 über eigene Abteilungen für Pathologie. Die Mehrheit der rund 1.500 Pathologen in Deutschland ist heutzutage in den etwa 300 freiberuflich organisierten Instituten tätig, die im Schnitt jeweils vier Krankenhäuser versorgen. Die flächendeckende Patientenversorgung wird durch die Tätigkeit mehrerer Fachärzte in den einzelnen Instituten sichergestellt, die gegebenenfalls mehrere Standorte abdecken können. Einzelpraxen sind die Ausnahme geworden.

Die Versorgung mehrerer Krankenhäuser bedeutet in der Regel auch, dass eine PathologieEinrichtung mit verschiedenen zertifizierten Organkrebszentren und/oder Onkologischen Zentren kooperiert. Die zu erfüllenden Anforderungen schließen das Vorhalten einer entsprechenden fachlichen Expertise, die Möglichkeit der Schnellschnittversorgung und die obligate Beteiligung an interdisziplinären Tumorkonferenzen ein. Berücksichtigt man die zunehmende Verknappung personeller Ressourcen, die ganz besonders auch die Pathologie betrifft, erscheint es naheliegend, auch in der Pathologie Möglichkeiten des Remote-Arbeitens einzuführen, um Fahrzeiten einzusparen, Personalressourcen zu bündeln und spezielle Fachexpertise schneller und niedrigschwelliger nutzen zu können (Farahani und Pantanowitz 2015; Griffin und Treanor 2017).

\subsubsection{Definition und Anwendungs- gebiete}

Die Ausübung der Pathologie über eine Entfernung unter Nutzung der Telekommunikation wird als Telepathologie bezeichnet. Dabei lassen sich die Erstellung einer Primärdiagnose (Telediagnose), beispielsweise auch im Kontext eines intraoperativen Schnellschnittes (Teleschnellschnitt), und die Zweitbegutachtung (Telekonsultation) unterscheiden. Außerdem kann die Telepathologie zur Qualitätssicherung, Konsensbildung, Fortbildung, Lehre, Forschung und in Telekonferenzen genutzt werden (Farahani und Pantanowitz 2015; Griffin und Treanor 2017). Gemeinsam ist diesen verschiedenen Optionen, dass das zu begutachtende Originalmaterial von den beurteilenden Pathologen räumlich getrennt ist.

\subsubsection{Technische Aspekte}

Die technischen Entwicklungen der letzten Jahre haben die Möglichkeiten der Telepathologie deutlich verbessert. Nachdem in den ersten Jahren nur Bilder von Präparateausschnitten per Video oder später digital versandt wurden (statische Telepathologie), eröffnete die Etablierung ferngesteuerter Mikroskope in den 1990er Jahren erstmals die Remote-Betrachtung vollständiger Schnittpräparate unter selbstständiger Auswahl relevanter Präparateausschnitte (dynamische Telepathologie). Seit Anfang der 2000er steht nun mit der virtuellen Mikroskopie eine Technik zur Verfügung, die es erlaubt, vollständige Schnittpräparate auf Glasobjektträgern in hoher Auflösung zeitlich und räumlich unabhängig vom Ort der Präparateherstellung zu begutachten (Farahani und Pantanowitz 2015; Griffin und Treanor 2017). In der Regel werden die gefärbten Schnittpräparate durch spezielle Mikroskope, sogenannte Slide-Scanner, vollautomatisiert mit einem 20x- oder 40x-Objektiv digitalisiert und je nach kommerziellem Anbieter in unterschiedlichen Bildformaten gespeichert. Die Bilder werden üblicherweise auf einem Server gespeichert, um einen Internet-basierten Zugang zu den Bildern zu ermöglichen. Der Server benötigt eine hohe Speicherkapazität, da die einzelnen Bilddateien mehrere 
Gigabytes (GB) groß sein können. Eine Visualisierung der Bilder über gängige Internet-Browser wird durch spezielle Software ermöglicht. Die virtuelle Mikroskopie stellt letztlich eine Art von „Streaming on Demand“ dar. Die Übertragungsgeschwindigkeit, die ein kritischer Faktor für die Routinetauglichkeit ist, hängt unter anderem von der Größe und der „Verkachelung“ der Bilddateien, der verwendeten Software, der Geschwindigkeit des Netzwerks und der Leistung des Endgeräts ab.

Als weitere technische Voraussetzungen für den Einsatz der Telepathologie sind das Einbinden der virtuellen Mikroskopie in das jeweils verwendete Pathologie-Informationssystem sowie das Vorhalten von Möglichkeiten der Telekommunikation zu nennen.

\subsubsection{Kosten}

Die Etablierung der virtuellen Mikroskopie, die auch als hochauflösende digitale Mikroskopie bezeichnet werden kann, ist außerordentlich kostenintensiv. Die Anschaffung von leistungsstarken, hochauflösenden Scannern und angemessenen Speichermedien sowie die notwendige Integration der Vor- und Nachbereitung des Scannens in den Workflow des Personals generiert Kosten, deren exakte Höhe von der Anzahl der zu scannenden Präparate abhängig ist und deshalb individuell variiert (Griffin und Treanor 2017). Die Kostenträger im deutschen Gesundheitswesen erstatten diesen Zusatzaufwand derzeit nicht im Rahmen der Patientenversorgung, sodass die Kosten von den Leistungserbringern allein getragen werden müssen.

\subsubsection{Rechtliche Situation und Qualitätssicherung}

Nach dem Grundsatz der Methodenfreiheit kann die Telepathologie zur pathomorphologischen Diagnostik eingesetzt werden. Prinzipiell gilt, dass der Einsatz telemedizinischer Technik die Rechtsverhältnisse nicht ändert (Dietel et al. 2000). Aus der Sorgfaltspflicht und der Gewährleistung des Facharztstandards ergibt sich die Verantwortung für den Einsatz der adäquaten technischen Mittel. Es muss sichergestellt sein, dass die übermittelten Daten und Bilder mit adäquaten technischen Mitteln erstellt, übermittelt und präsentiert werden. Außerdem ist zu gewährleisten, dass die digitalisierten Bilder am Bildschirm mit der gleichen diagnostischen Treffsicherheit beurteilt werden können wie mit dem herkömmlichen Verfahren (Dietel et al. 2000; Hufnagl et al. 2018a). Eine Hilfestellung bei der Qualitätssicherung und der Validierung bietet der kürzlich publizierte Leitfaden Digitale Pathologie (Hufnagl et al. 2018a; Hufnagl et al. 2018b).

Es besteht eine Dokumentationspflicht wie bei der konventionellen Diagnostik. Das Auseinanderfallen von Handlungs- und Erfolgsort kann dazu führen, dass bei internationaler Nutzung deutsches und ausländisches Recht konkurrieren. Diese potenziellen Risiken sind bei der Implementierung ebenso wie mögliche technische, finanzielle und sprachliche Barrieren zu bedenken (Farahani et al. 2016).

\subsubsection{Möglichkeiten und Grenzen}

Die Beantwortung der Frage, ob und wie Telepathologie in der täglichen Routine der onkologischen Diagnostik von Nutzen ist, bedarf einer differenzierten Betrachtung der Möglichkeiten und Grenzen in den verschiedenen diagnostischen Szenarien. Studien zur diagnostischen Qualität zeigen mehrheitlich eine hohe Konkordanz zwischen Telepathologie und konventioneller Lichtmikroskopie. Dies gilt insbesondere für die Diagnose von kolorektalen Karzinomen und Mammakarzinomen. Schwierigkeiten kann allerdings die Begutachtung nukleärer oder zellulärer Details bereiten, die sich beim Grading oder in der Zytopathologie bemerkbar machen können (Bashshur et al. 2017). Im Einzelnen ergeben sich für die verschiedenen Remote-Szenarien folgende Vorteile und Limitationen:

\section{- Primärdiagnostik (Telediagnostik, Teleschnellschnitt)}

Die Nutzung der virtuellen Mikroskopie von Paraffinschnitten zur Primärdiagnostik in größeren Pathologie-Einrichtungen, die an mehreren Standorten tätig sind, ermöglicht eine Zentralisierung der Gewebe- und Schnittpräparation unter Einsparung 
der Transportwege für die erstellten Schnittpräparate zum entfernten Standort. Dies erlaubt eine Bündelung personeller Ressourcen und eine größere räumliche und zeitliche Flexibilität (Griffin und Treanor 2017). Die Kombination mit weiteren digitalen Werkzeugen (Barcodes auf Probengefäßen, Gewebeblöcken und Schnittpräparaten, digitales Diktieren) kann die Sicherheit und Effizienz der Diagnostik verbessern. Diese Vorteile sind gegenüber den Kosten und dem Zeitaufwand abzuwägen, die das Digitalisieren der Schnittpräparate und der geänderte Workflow bedingen. Gerade bei onkologischen Fällen ist die Anzahl der anfallenden Schnittpräparate in der Regel hoch, da neben der konventionellen Histologie noch Zusatzuntersuchungen (Immunhistologie, Molekularpathologie) erforderlich sein können.

Es erscheint naheliegend, die Telepathologie zu nutzen, um die Schnellschnittdiagnostik auch an Standorten ohne lokal verfügbaren Pathologen anbieten zu können. In bevölkerungsarmen Regionen anderer Länder wird dies bereits seit längerem praktiziert (Huang et al. 2018; Perron et al. 2014; Tetu et al. 2014). Andernfalls besteht die Notwendigkeit, das Gewebe zur Pathologie zu transportieren oder die Pathologen und das erforderliche technische Personal für die Schnellschnittdiagnostik zum Standort zu bringen. In Deutschland ist zu bedenken, dass der Pathologe gegenüber dem Patienten für seine Diagnose haftbar ist, unabhängig vom $\mathrm{Ab}$ lauf der pathologischen Diagnostik. Alle Schritte, die zur Diagnose führen, müssen in der Verantwortung des Pathologen verbleiben: Zuschnitt, Anfertigung der mikroskopischen Schnittpräparate, deren Digitalisierung, Übertragung und Begutachtung. Wird das Präparat für den Schnellschnitt vom Operateur und nicht vom Pathologen zugeschnitten, so handelt es sich für den Operateur um eine fachfremde Leistung. Dies gilt auch für den Fall, dass der Operateur per Makrokamera und Telekommunikation von dem Pathologen angeleitet wird. Dementsprechend ist diese Einsatzform der Telepathologie aus Sicht des Patientenschutzes außerhalb von Studien in Deutschland unzulässig. Im Einzelnen wird gegen verschiedene rechtliche Regelungen verstoßen: Weiterbildungsordnung, Berufsordnung, Heilberufe-Gesetz, Gebührenrecht und Haftungsrecht. Darüber hinaus besteht die Aufklärungs- pflicht, den Patienten in Kenntnis zu setzen, dass der Teleschnellschnitt gegen fachärztliche Routine verstößt.

Zudem ist anzumerken, dass die Teleschnellschnittdiagnostik gemäß den Anforderungen der DIN EN ISO/IEC 17020:2012 nicht akkreditierbar ist, wenn kein Inspektor (Pathologe) am Ort des Schnellschnitts anwesend ist (Deutsche Akkreditierungsstelle 2017).

\section{- Zweite Meinung (Telekonsultation)}

Das Einholen einer Zweitmeinung unter Nutzung der Telepathologie kann die Qualität und die Schnelligkeit der Diagnostik verbessern (Bashshur et al. 2017; Perron et al. 2014; Romero Lauro et al. 2013). Dies gilt insbesondere für interne Konsile, die innerhalb einer Institution eingeholt werden, die auf verschiedene Standorte verteilt ist. Durch das schnelle, niedrigschwellige Einholen einer internen Zweitmeinung bei schwierigen Fällen können Zusatzuntersuchungen gezielter eingesetzt, das Risiko einer Fehlentscheidung verringert und die Anzahl externer Konsile reduziert werden. Gegenstand solcher interner Telekonsile können auch Schnellschnitte sein, bei denen sich der Pathologe in der Außenstelle den Rat eines Kollegen im Kerninstitut holt.

Für das formale Einholen einer externen Zweitmeinung gibt es verschiedene Gründe: die Bitte des primär beauftragten Pathologen um die Einschätzung eines Experten bei schwierigen oder seltenen Fällen, der Wunsch des Patienten oder die Weiterbehandlung eines Patienten an einer anderen Institution. Bei Nutzung der Telepathologie entfallen der traditionelle Versand der Schnittpräparate und damit auch das Risiko des Verlusts oder der Beschädigung. Dies gilt allerdings nur für die Fälle, in denen der Konsilpathologe anhand der telepathologisch zur Verfügung gestellten digitalisierten Schnittpräparate zu einer abschließenden Einschätzung kommen kann. Sind aus seiner Sicht aber Zusatzuntersuchungen notwendig, die möglicherweise vom Primärpathologen nicht bedacht wurden oder auch nicht dort etabliert sind, kann der Fall nicht abgeschlossen werden und es ist gegebenenfalls doch ein Blockversand zur Komplettierung notwendig. Zu bedenken ist außerdem, dass gerade bei schwierigen, komplexen Fällen mit vielen $\mathrm{Zu}$ - 
satzuntersuchungen der Aufwand für das Digitalisieren der Objektträger erhöht sein kann. Das Haftungsrisiko liegt bei der Telekonsultation ebenso wie bei der traditionell eingeholten Zweitmeinung im Wesentlichen beim primär beauftragten Pathologen, der auch für die Qualität der zu begutachtenden virtuellen Präparate verantwortlich ist.

\subsection{Zusammenfassung und Ausblick}

Die Etablierung von Remote-Arbeitsplätzen erlaubt eine höhere zeitliche und räumliche Flexibilität der beteiligten Pathologen. Die Nutzung der Telepathologie kann zudem das Einholen einer Zweitmeinung erleichtern und hierdurch die Qualität und Geschwindigkeit der Diagnostik steigern. Der Einsatz der virtuellen Mikroskopie zur Standardisierung von Fortbildungen trägt ebenso zur Qualitätsverbesserung bei. Limitiert wird die Nutzung der Telepathologie insbesondere durch die hohen nicht erstattungsfähigen Kosten sowie Haftungsfragen, die einer gesonderten Betrachtung bedürfen. Wünschenswert wäre eine Vereinheitlichung der Standards der Telepathologiesysteme, damit eine Kommunikation der Systeme untereinander gewährleistet ist.

\subsection{Diskussion}

Trotz der zunehmenden Digitalisierung unserer Gesellschaft und des - auch politisch gewollten zunehmenden Einsatzes moderner IKT ist die Einführung von Telemedizin im Gesundheitswesen kein Selbstzweck. Jedes telemedizinische Versorgungskonzept sollte vor Implementierung gründlich auf sein Ziel, seinen Nutzen und seine Aufwände geprüft werden. Ein besonders wichtiges Kriterium ist die Beibehaltung oder Verbesserung der Versorgungsqualität.

Auch in der Onkologie spielt die Telemedizin eine zunehmend wichtige Rolle. Da die Struktur der zertifizierten Zentren der DKG ein hohes Maß an interdisziplinärer Zusammenarbeit und Kommunikation erforderlich macht und zugleich die Einbringung verschiedenster onkologischer Expertise in das Versorgungsnetzwerk fordert, können tele- medizinische Lösungen ein gut geeignetes Instrument sein, um in der Behandlung von Krebspatienten Synergien zu schaffen, Austausch zu fördern und Versorgungsdefizite zu adressieren. Dies trifft insbesondere auf telemedizinische Anwendungen für die Arzt-zu-Arzt-Kommunikation zu, wie die Beispiele der Tele-Tumorkonferenz und der Telepathologie illustrieren. Damit die Versorgungsqualität bei der digitalen Kommunikation erhalten bleibt, müssen aber auch einige Voraussetzungen erfüllt sein. Relevant für eine störungsfreie Kommunikation ist mitunter, dass die verwendeten Applikationen bedienerfreundlich sind und technisch einwandfrei funktionieren. Auch rechtliche Fragestellungen sind kritisch zu betrachten und können, beispielsweise wenn die Telemedizin Einbußen beim Facharztstandard bedingt, unmittelbaren Einfluss auf die Qualität der Patientenversorgung haben. Nicht zuletzt zeigen sowohl die Tele-Tumorkonferenz als auch die Telepathologie, dass die Implementierung von Telemedizin mit erheblichem finanziellem und organisatorischem Aufwand verbunden ist, der insbesondere bei fehlender Vergütung durch die Leistungsträger gegenzurechnen ist.

Noch sorgfältiger sollten telemedizinische Anwendungen für die Arzt-zu-Patienten-Kommunikation vor ihrer Einführung in der onkologischen Versorgung auf den Prüfstand gestellt werden. Da die Diagnose Krebs bei den Patienten Sorgen und Ängste auslöst und sie zugleich mit komplexen, weichenstellenden Fragen zu Diagnostik und Therapie konfrontiert werden, ist die Kommunikation zwischen Arzt und Patient ein zentrales Element der onkologischen Versorgung. Die Erzielung hoher Versorgungsqualität ist von einem vertrauensvollen Verhältnis zwischen Patient und Leistungserbringer sowie einer Kommunikation frei von Missverständnissen und ohne Informationsverluste abhängig. Ob die Interaktion mit modernen IKT diese Standards gewährleisten kann, ist fraglich. Studien, die den Nutzen telemedizinischer Interventionen zur Interaktion zwischen Patienten und Leistungserbringern im onkologischen Versorgungskontext untersuchen, zeigen heterogene Ergebnisse (Slev et al. 2016). In einigen Studien weisen telemedizinische Versorgungsangebote keine Vorteile gegenüber der Standardversorgung auf oder können sogar mit Nachteilen hinsichtlich patientenrelevanter Para- 
meter verbunden sein (Hoek et al. 2017; Hass et al. 2017). Die heterogene Studienlage unterstreicht, dass noch Forschungsbedarf zu der Frage besteht, unter welchen Rahmenbedingungen und in welcher Form die Telemedizin in der Kommunikation zwischen Arzt und Krebspatient sinnvoll eingesetzt werden kann.

\subsection{Fazit}

Bereits heute sind telemedizinische Strukturen etablierter Bestandteil der onkologischen Versorgung, beispielsweise in den Bereichen der Tumorkonferenzen, der Radiologie und der Pathologie. Bedingt durch die gesellschaftliche und gesundheitspolitische Entwicklung ist mit einem weiteren Ausbau der Telemedizin zu rechnen. Die Potenziale der Telemedizin müssen dabei immer so eingesetzt werden, dass sie fördern, worauf es für die Krebspatienten und die Qualität ihrer Behandlung ankommt: die Einbringung fachlicher Expertise, der interdisziplinäre Austausch der Leistungserbringer, ein vertrauensvolles Behandlungsumfeld und eine Versorgung nach dem aktuellen medizinischen Kenntnisstand.

\section{Literatur}

Bashshur RL, Krupinski EA, Weinstein RS, Dunn MR, Bashshur N (2017) The Empirical Foundations of Telepathology: Evidence of Feasibility and Intermediate Effects. Telemed J E Health 23(3):155-191

Breuer L, Schwab S (2017) Telemedizin in der Schlaganfallversorgung. Med Klin Intensivmed Notfmed 112(8):687-694

Bundesärztekammer (2015) Ärztliche Positionen zu Einsatzgebieten telemedizinischer Patientenversorgung. http:// www.bundesaerztekammer.de/aerzte/telematiktelemedizin/telemedizin/. Zugegriffen: 27. Juni 2018

Bundesärztekammer (2018) (Muster-)Berufsordnung für die in Deutschland tätigen Ärztinnen und Ärzte in der Fassung des Beschlusses des 121. Deutschen Ärztetages 2018 in Erfurt. Dtsch Ärztebl 115(24):A1190

Bundesministerium für Wirtschaft und Energie (2016) Ökonomische Bestandsaufnahme und Potenzialanalyse der digitalen Gesundheitswirtschaft. Bundesministerium für Wirtschaft und Energie, Berlin

Deutsche Akkreditierungsstelle (2017) Anforderungen der DIN EN ISO/IEC 17020:2012 und technische Kriterien für deren Anwendung zur Akkreditierung in der Pathologie/ Neuropathologie. 71 SD 4.001, Revision 1.6. https://www. dakks.de/content/anforderungen-der-din-en-isoiec-
170202012-und-technische-kriterien-f\%C3\%BCr-derenanwendung-zur-a. Zugegriffen: 19 Juli 2018

Deutscher Bundestag (2015) Gesetz für sichere digitale Kommunikation und Anwendungen im Gesundheitswesen sowie zur Änderung weiterer Gesetze. Bundesgesetzblatt Jahrgang 2015 Teil I Nr. 45, S 2408-2423

Dietel M, Hufnagl P, Schlake W, Bollmann R, Dettmar P, Hofler $\mathrm{H}$ et al (2000) Arbeitspapier zur Telepathologie Version 2. Bundesverband Deutscher Pathologen, Berlin

Farahani N, Pantanowitz L (2015) Overview of Telepathology. Surg Pathol Clin 8(2):223-231

Farahani N, Riben M, Evans AJ, Pantanowitz L (2016) International Telepathology: Promises and Pitfalls. Pathobiology 83(2-3):121-126

Fischer F, Aust V, Krämer A (2016) eHealth: Hintergrund und Begriffsbestimmung. In: Fischer F, Krämer A (Hrsg) eHealth in Deutschland. Springer, Berlin Heidelberg, S 3-23

Griffin J, Treanor D (2017) Digital pathology in clinical use: where are we now and what is holding us back? Histopathology 70(1):134-145

Güttler FV, Rakowsky S, Nagel SN, Teichgräber U, Schlag PM (2012) Interdisziplinäre Tumorkonferenzen. Der Onkologe 18(5):389-397

Hass HG, Muthny F, Stepien J, Lerch J, von der Marwitz C, Schrock R et al (2017) Effekte der telefonischen Nachsorge in der onkologischen Rehabilitation nach Brustkrebs Ergebnisse einer randomisierten Studie. Rehabilitation (Stuttg) 56(3):189-197

Hoek PD, Schers HJ, Bronkhorst EM, Vissers KCP, Hasselaar JGJ (2017) The effect of weekly specialist palliative care teleconsultations in patients with advanced cancer a randomized clinical trial. BMC Med 15(1):119

Huang Y, Lei Y, Wang Q, Li D, Ma L, Guo L et al (2018) Telepathology consultation for frozen section diagnosis in China. Diagn Pathol 13(1):29

Hufnagl P, Zwönitzer R, Haroske G (2018a) Leitfaden Digitale Pathologie in der Diagnostik - Befunderstellung an digitalen Bildern Version 1.0. Bundesverband Deutscher Pathologen, Berlin

Hufnagl P, Lohmann S, Schluns K, Zerbe N (2018b) Umsetzung des Leitfadens "Digitale Pathologie in der Diagnostik" - Unterstützende Systeme und ihre Funktionalität. Pathologe 39(3):222-227

Janssen A, Brunner M, Keep M, Hines M, Nagarajan SV, KiellyCarroll C et al (2017) Interdisciplinary eHealth Practice in Cancer Care: A Review of the Literature. Int J Environ Res Public Health 14(11)

Kowalski C, Graeven U, von Kalle C, Lang H, Beckmann MW, Blohmer JU et al (2017) Shifting cancer care towards Multidisciplinarity: the cancer center certification program of the German cancer society. BMC Cancer 17(1):850

Marx G, Beckers R (2015) Telemedizin in Deutschland. Bundesgesundheitsbl 58:1053-1055

Mensah J, Graeven U, Wesselmann S (2017) Nationales Zertifizierungsprogramm Krebs. Der Onkologe 23(9): $711-718$ 
Perron E, Louahlia S, Nadeau L, Boilard F, Ing M, Orain M et al (2014) Telepathology for intraoperative consultations and expert opinions: the experience of the Eastern Quebec Telepathology Network. Arch Pathol Lab Med 138(9):1223-1228

Robert Koch-Institut (2017) Krebs in Deutschland für 2013/2014. Robert Koch-Institut, Berlin

Romero Lauro G, Cable W, Lesniak A, Tseytlin E, McHugh J, Parwani A et al (2013) Digital pathology consultations-a new era in digital imaging, challenges and practical applications. J Digit Imaging 26(4):668-677

Schenkel J (2017) Praxis der Telemdizin. Dtsch Med Wochenschr 142:328-333

Slev VN, Mistiaen P, Pasman HR, Verdonck-de Leeuw IM, van Uden-Kraan CF, Francke AL (2016) Effects of eHealth for patients and informal caregivers confronted with cancer: A meta-review. Int J Med Inform 87:54-67
Tetu B, Perron E, Louahlia S, Pare G, Trudel MC, Meyer J (2014) The Eastern Quebec Telepathology Network: a three-year experience of clinical diagnostic services. Diagn Pathol 9 Suppl 1:S1

Trautmann F, Reissfelder C, Pecqueux M, Weitz J, Schmitt J (2018) Evidence-based quality standards improve prognosis in colon cancer care. Eur J Surg Oncol

Vesta Informationsportal (2018) https://www.informationsportal.vesta-gematik.de/ Zugegriffen: 27. Juni 2018

Völkel V, Draeger T, Gerken M, Fürst A, Klinkhammer-Schalke M (2018) Langzeitüberleben von Patienten mit Kolonund Rektumkarzinomen: Ein Vergleich von Darmkrebszentren und nicht zertifizierten Krankenhäusern. Gesundheitswesen (EFirst)

Wesselmann S (2012) Entwicklung der Zertifizierungsverfahren für Organkrebszentren und Onkologische Zentren der Deutschen Krebsgesellschaft. Der Onkologe 18(6):511-516

Open Access Dieses Kapitel wird unter der Creative Commons Namensnennung 4.0 International Lizenz(http://creativecommons. org/licenses/by/4.0/deed.de) veröffentlicht, welche die Nutzung, Vervielfältigung, Bearbeitung, Verbreitung und Wiedergabe in jeglichem Medium und Format erlaubt, sofern Sie den/die ursprünglichen Autor(en) und die Quelle ordnungsgemäß nennen, einen Link zur Creative Commons Lizenz beifügen und angeben, ob Änderungen vorgenommen wurden.

Die in diesem Kapitel enthaltenen Bilder und sonstiges Drittmaterial unterliegen ebenfalls der genannten Creative Commons Lizenz, sofern sich aus der Abbildungslegende nichts anderes ergibt. Sofern das betreffende Material nicht unter der genannten Creative Commons Lizenz steht und die betreffende Handlung nicht nach gesetzlichen Vorschriften erlaubt ist, ist für die oben aufgeführten Weiterverwendungen des Materials die Einwilligung des jeweiligen Rechteinhabers einzuholen. 